\title{
Self-organized generation of transverse waves in diverging cylindrical detonations
}

\author{
Zonglin Jiang $^{\mathrm{a}, *, 1}$, Guilai Han ${ }^{\mathrm{a}}$, Chun Wang ${ }^{\mathrm{a}, 2}$, Fan Zhang ${ }^{\mathrm{b}}$ \\ ${ }^{a}$ Key Laboratory of High Temperature Gas Dynamics, Institute of Mechanics, Chinese Academy of Sciences, Beijing 100190, China \\ ${ }^{\mathrm{b}}$ Defence Research and Development Canada-Suffield, P.O. Box 4000, Stn. Main, Medicine Hat, Canada AB T1A8K6
}

\section{A R T I C L E I N F O}

\section{Article history:}

Received 4 November 2008

Received in revised form 23 February 2009

Accepted 27 February 2009

Available online 20 May 2009

\section{Keywords:}

Cylindrical detonation

Transverse waves

Cellular detonation instabilities

Cell bifurcation

Numerical simulation

\begin{abstract}
A B S T R A C T
Self-organized generation of transverse waves associated with the transverse wave instabilities at a diverging cylindrical detonation front was numerically studied by solving two-dimensional Euler equations implemented with an improved two-step chemical kinetic model. After solution validation, four mechanisms of the transverse wave generation were identified from numerical simulations, and referred to as the concave front focusing, the kinked front evolution, the wrinkled front evolution and the transverse wave merging, respectively. The propagation of the cylindrical detonation is maintained by the growth of the transverse waves that match the rate of increase in surface area of the detonation front to asymptotically approach a constant average number of transverse waves per unit length along the circumference of the detonation front. This cell bifurcation phenomenon of cellular detonations is discussed in detail to gain better understanding on detonation physics.
\end{abstract}

( 2009 The Combustion Institute. Published by Elsevier Inc. All rights reserved.

\section{Introduction}

In unconfined cylindrical and spherical geometries, the surface area of the detonation front continuously increases with radius as a diverging detonation wave expands. The propagation mechanism of a diverging detonation wave remains a subject of research in the detonation theory. Theoretical studies of diverging cylindrical and spherical detonation and its steady solution originate in the pioneer works of Jouguet [1], Courant and Friedrich [2], Taylor [3], Manson and Ferrie [4], and Lee et al. [5]. Assuming a steady C-J detonation where the product flow is isentropic if no further chemical reactions, frictions and heat losses are considered, the basic isentropic equations can be expressed by

$$
\begin{aligned}
& \frac{2}{\gamma-1} \frac{\partial c}{\partial t}+\frac{2}{\gamma-1} u \frac{\partial c}{\partial r}+c \frac{\partial u}{\partial r}+\frac{j c u}{r}=0 \\
& \frac{\partial u}{\partial t}+u \frac{\partial u}{\partial r}+\frac{2}{\gamma-1} c \frac{\partial c}{\partial r}=0
\end{aligned}
$$

where $j=1$ or 2 denotes cylindrical or spherical symmetry, respectively. Assuming central ignition at $r=0$ without any length scales, the solution is self-similar in the variable $\xi=r / D t$ where $D$ is the steady $\mathrm{C}-\mathrm{J}$ detonation speed. Upon non-dimensionalizing the dependant variables by $\phi(\xi)=u / D$ and $\beta(\xi)=c / D$ in Eqs. (1) and (2), one obtains

\footnotetext{
* Corresponding author. Fax: +86 01082543996.

E-mail addresses: zljiang@imech.ac.cn (Z. Jiang), hangulai@imech.ac.cn (G. Han), wangchun@imech.ac.cn (C. Wang), fanchyang@hotmail.com (F. Zhang).

1 Visiting Professor at McGill University, Montreal, Canada.

2 Fax: +86010 82543996 .
}

$$
\begin{aligned}
\frac{d \phi}{d \xi} & =\frac{j \phi \beta^{2}}{\xi} \frac{1}{(\phi-\xi)^{2}-\beta^{2}} \\
\frac{d \beta}{d \xi} & =-\frac{\gamma-1}{2} \frac{j \phi \beta(\phi-\xi)}{\xi} \frac{1}{(\phi-\xi)^{2}-\beta^{2}} .
\end{aligned}
$$

At the detonation front where $\xi=1$, the $\mathrm{C}-\mathrm{J}$ criterion requires

$D=u+c$ or $(\phi-\xi)^{2}-\beta^{2}=0$

For cylindrical or spherical detonation waves for which $j \neq 0$, the singularity at the front is apparent in Eqs. (3) and (4), and the question on the existence of the diverging C-J detonation arises. Strictly speaking, such an infinite expansion gradient behind the wave front will make expansion waves penetrate into the reaction zone and attenuate detonation waves rapidly. Thus, a rigorous steady solution of diverging cylindrical or spherical detonation under point initiation does not exist. Taylor [3] argued that the error is likely to be of the order of the ratio of the thickness of the reaction zone to the radius of the detonation front and diminishes as the detonation wave expands. Lee [5] stated that high initiation energy must be used and overdrives the initial detonation to overcome the initially large diverging expansion rate.

The curvature of the detonation front essentially has a destabilizing effect on detonation. Therefore, the diverging cylindrical or spherical detonation may no longer be a C-J steady detonation. The smaller the propagation radius is, the larger the front curvature and hence more unstable the detonation is. In order to take into account the influence of diverging detonation front curvature, a "generalized C-J condition", originating in the work of Wood and Kirkwood [6] and Zeldovich [7], may be considered to replace the 
classic C-J condition. This leads to a quasi-steady solution asymptotically approaching the $\mathrm{C}-\mathrm{J}$ steady detonation in the far-field. The quasi-steady solution appears to agree with the mean value of the asymptotic solution of one-dimensional unsteady numerical simulations of directly initiated (initially overdriven) diverging detonations [8-12]. A decrease in direct initiation energy results in an increase in initial unstable detonation behavior (e.g., amplitude and growth rate of the detonation oscillation). Watt and Sharpe further showed that it is feasible to initiate a diverging detonation wave using a quasi-steady solution domain, where the initial detonation behavior becomes more unstable with a decrease in initial quasi-steady solution domain. Preliminary two-dimensional numerical studies of detonation instabilities have also been conducted for diverging cylindrical detonation waves [13]. The splitting and merging of the cells in cylindrical detonation have been primarily investigated by Han et al. [14].

Experimentally, Soloukhin $[15,16]$ reported a clean open-shuttle photograph of a diverging cylindrical detonation as shown in Fig. 1 . The detonation was initiated in a circular tube and radially expanded in an adjoining cylindrical disk. Soloukhin [16] stated: "A series of luminous points, corresponding to two systems of acoustic waves traveling in opposite directions, move transversely through the radialy expanding detonation front. The trajectories of these luminous points are imprinted on the fixed film. The transverse fronts are generated in such a way that the average distance between adjacent perturbations hardly varies, except during the initial stage of the process". In this statement he highlighted that the detonation propagation mechanism is likely controlled by the transverse wave instabilities, and that there appears a detonation front structure with transverse wave spacing asymptotically towards a constant size while the front surface increases continuously. But he simplified transverse wave instabilities into two acoustic wave trajectory systems in logarithmic spirals that cannot explain the nearly constant transverse wave spacing observed as the circumference of the detonation front increases with the expanding detonation. Diverging spherical detonation experiments can also be found in the literature $[17,18]$. These experiments were mainly concerned with the critical energy required for initiating detonation and the obtained detonation velocity asymptotically approaches a constant. However, there was no conclusion for the propagation mechanism due to the limited information obtained.

The main purpose of this paper is to study transverse wave instabilities in which the propagation mechanism of the diverging

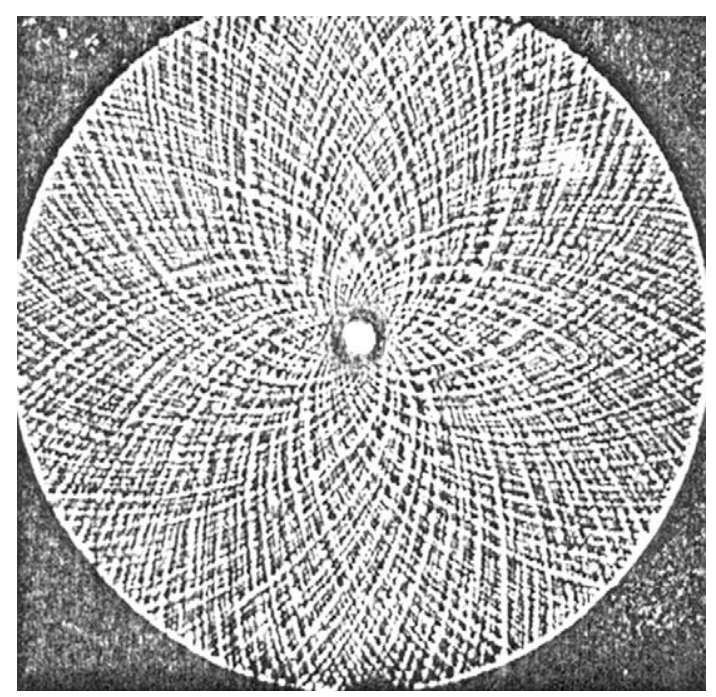

Fig. 1. Open-shuttle photograph of a diverging cylindrical detonation in $\mathrm{C}_{2} \mathrm{H}_{2}-\mathrm{O}_{2}$ mixture at initial pressure of $60 \mathrm{mmHg}[16]$. cylindrical or spherical detonation could reside. As the diverging detonation wave expands, there must be new transverse waves inherently generated on the enlarging detonation front to form new cells asymptotically towards a constant cell size pattern. The origin and the mechanism of this self-organized generation of transverse waves will be investigated using two-dimensional unsteady numerical simulations to gain sufficient mesh resolution. The conclusion may be helpful to understand three-dimensional spherically-diverging detonation since a similar nature of detonation diverging exists between the spherical and cylindrical detonations.

\section{Physical description and computational domain}

A simple physical domain for the diverging cylindrical detonation is schematically shown in Fig. 2a, where $R$ is the radius of the physical domain filled with a detonable gaseous mixture at a given initial temperature $T_{0}$, pressure $p_{0}$ and flow velocity $v_{0}$. The central ignition source is taken to be a hot spot of burned gas ( $p=5.0 \mathrm{MPa}$ and $T=3000 \mathrm{~K}$ within a circle layer of 2 times the half reaction zone) for a direct initiation of cylindrical detonation with the minimum initial overdriven effect.

In numerical experiments, a stoichiometric hydrogen-oxygen mixture is filled into the physical domain at the initial state of $p_{0}=1.0 \mathrm{~atm}$ and $T_{0}=293.0 \mathrm{~K}$. By assuming flow symmetry, the computational domain is simplified as a $30^{\circ}$ annular region, as shown in Fig. $1 \mathrm{~b}$, with an outer radius of $15.5 \mathrm{~cm}$ and an inner radius of $0.5 \mathrm{~cm}$ to avoid the singularity problem. In the computational domain, the orthogonal mesh system in the cylindrical coordinates is applied; 3000 grid points are uniformly distributed in the radial direction and 1000 grid points in the circumferential direction. Mesh resolution tests were conducted up to 30 grid points per half reaction zone, and about 15 grid points per half reaction zone is finally chosen for the calculations, and more details can be found in the following section for the validation of
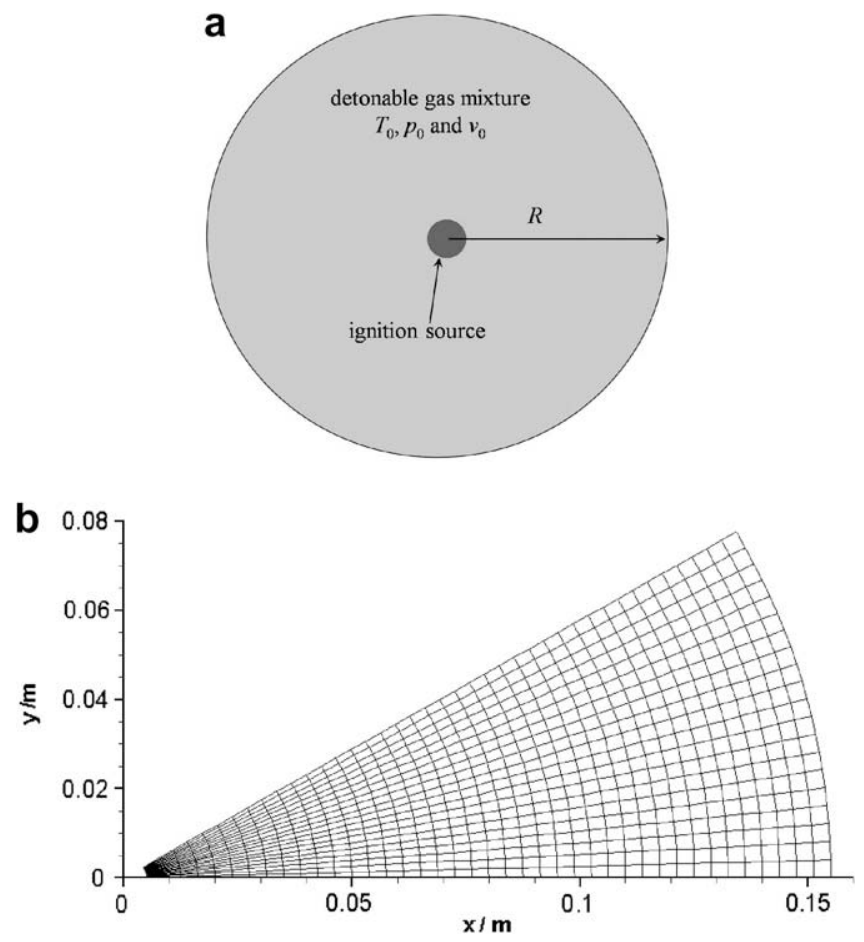

Fig. 2. (a) Problem specifications and the physical domain for a diverging cylindrical detonation; (b) the 30-degree angular computational domain with an orthogonal grid mesh in cylindrical coordinate. 
numerical methods and code. Although an ultimate solution for the detonation instabilities requires much finer mesh resolution, the grid chosen is possible to qualitatively elucidate the influence of the instability and generation of transverse waves on the maintenance of diverging detonation propagation.

\section{Governing equations and chemical kinetic model}

Assuming that the effect of viscosity on the dynamic process of detonation propagations is negligible and the flow field of interest is of cylindrical symmetry, the governing equations for detonations can be simplified as the two-dimensional reactive Euler equations in Cartesian coordinate system:

$\frac{\partial \mathbf{U}}{\partial t}+\frac{\partial \mathbf{F}}{\partial x}+\frac{\partial \mathbf{G}}{\partial y}=\mathbf{S}$

$\mathbf{U}=\left[\begin{array}{c}\rho \alpha \\ \rho \beta \\ \rho \\ \rho u \\ \rho v \\ E\end{array}\right], \quad \mathbf{F}=\left[\begin{array}{c}\rho u \alpha \\ \rho u \beta \\ \rho u \\ \rho u^{2}+p \\ \rho u v \\ (E+p) u\end{array}\right], \quad \mathbf{G}=\left[\begin{array}{c}\rho v \alpha \\ \rho v \beta \\ \rho v \\ \rho u v \\ \rho v^{2}+p \\ (E+p) v\end{array}\right], \quad \mathbf{S}=\left[\begin{array}{c}\rho \dot{\omega}_{\alpha} \\ \rho \dot{\omega}_{\beta} \\ 0 \\ 0 \\ 0 \\ 0\end{array}\right]$

where the variable $E$ stands for the total energy per specific volume and $p$ is the sum of the partial pressure for each species according to Dalton's law with the ideal gas equation of state. Variables $\alpha$ and $\beta$ specify the degree of chemical induction and heat release reaction of an improved two-step kinetics model proposed by Sichel et al. [19]. In this model, the change of chemical components during reactions is included in the calculation of thermodynamic parameters and the reaction rates of the two-step chemical processes are expressed by

$$
\left\{\begin{array}{l}
\dot{\omega}_{\alpha}=\frac{P}{3 R_{0} T} \exp \left[35.1715-\frac{8530.6}{T}-7.22 \times 10^{-11} P^{2} \exp \left(\frac{21205}{T}\right)\right] \\
\dot{\omega}_{\beta}=a(1-\beta) \exp \left(-\frac{b}{T}\right)+c
\end{array}\right.
$$

where $a=1.2 \times 10^{8}, b=8.0 \times 10^{8}, c=0$ and the unit of pressure $P$ is atm. For chemical reactions, all species are assumed to be thermally perfect gases; the specific heats at constant pressure $c_{p i}$ and the specific enthalpies $h_{i}$ for each species are calculated with the fitting functions expressed as [20]

$$
\left\{\begin{array}{l}
\frac{c_{p i}}{R_{i}}=a_{1 i} \frac{1}{T^{2}}+a_{2 i} \frac{1}{T}+a_{3 i}+a_{4 i} T+a_{5 i} T^{2}+a_{6 i} T^{3}+a_{7 i} T^{4} \\
\frac{h_{i}}{R_{i} T}=-a_{1 i} \frac{1}{T^{2}}+a_{2 i} \frac{1}{T} \ln T+a_{3 i}+\frac{a_{4 i}}{2} T+\frac{a_{5 i}}{3} T^{2}+\frac{a_{6 i}}{4} T^{3}+\frac{a_{7 i}}{5} T^{4}+\frac{b_{1 i}}{T}
\end{array}\right.
$$

For a steady planar ZND detonation, the length ratio of induction zone to heat release reaction zone is about $0.1-0.3$ according to Eq. (8). The mesh resolution (15 point within the half reaction zone length) is referred to the sum of the induction and heat release zones of the steady ZND detonation following this two-step model.

Upon a general coordinate transformation, the governing equations in computational space are written as

$\frac{\partial \widetilde{\mathbf{U}}}{\partial \tau}+\frac{\partial \widetilde{\mathbf{F}}}{\partial \xi}+\frac{\partial \widetilde{\mathbf{G}}}{\partial \eta}=\widetilde{\mathbf{S}}$

with

$$
\begin{aligned}
& \widetilde{\mathbf{U}}=\frac{\mathbf{U}}{J}, \quad \widetilde{\mathbf{E}}=\frac{1}{J}\left(\xi_{x} \mathbf{F}+\xi_{y} \mathbf{G}\right), \quad \widetilde{\mathbf{F}}=\frac{1}{J}\left(\eta_{x} \mathbf{F}+\eta_{y} \mathbf{G}\right), \\
& \widetilde{\mathbf{S}}=\frac{\mathbf{S}}{J} \quad \text { and } \quad J=\frac{\partial(\xi, \eta)}{\partial(x, y)}
\end{aligned}
$$

where $J$ is the Jacobian of transformation.
In order to capture shock waves efficiently, the NND scheme is adopted to discrete two-dimensional governing equations [21], and the obtained semi-discreted finite difference equations are written as

$\frac{\partial \widetilde{\mathbf{U}}_{i, j}}{\partial \tau}=-\frac{1}{\Delta \xi}\left(\overline{\mathbf{F}}_{i+\frac{1}{2}, j}-\overline{\mathbf{F}}_{i-\frac{1}{2}, j}\right)-\frac{1}{\Delta \eta}\left(\overline{\mathbf{G}}_{i, j+\frac{1}{2}}-\overline{\mathbf{G}}_{i, j-\frac{1}{2}}\right)+\widetilde{\mathbf{S}}_{i, j}$

with

$\left\{\begin{array}{l}\overline{\mathbf{F}}_{i+\frac{1}{2}, j}=\widetilde{\mathbf{F}}_{i+\frac{1}{2} L, j}^{+}+\widetilde{\mathbf{F}}_{i+\frac{1}{2} R, j}^{-} \\ \overline{\mathbf{G}}_{i, j+\frac{1}{2}}=\widetilde{G}_{i, j+\frac{1}{2} L}^{+}+\widetilde{\mathbf{G}}_{i, j+\frac{1}{2} R}^{-}\end{array}\right.$

$\left\{\begin{array}{l}\widetilde{\mathbf{F}}_{i+\frac{1}{2} L, j}^{+}=\widetilde{\mathbf{F}}_{i, j}^{+}+\frac{1}{2} \min \bmod \left(\widetilde{\mathbf{F}}_{i, j}^{+}-\widetilde{F}_{i-1, j}^{+}, \widetilde{\mathbf{F}}_{i+1, j}^{+}-\widetilde{\mathbf{F}}_{i, j}^{+}\right) \\ \widetilde{\mathbf{G}}_{i, j+\frac{1}{2} L}^{+}=\widetilde{\mathbf{G}}_{i, j}^{+}+\frac{1}{2} \min \bmod \left(\widetilde{\mathbf{G}}_{i, j}^{+}-\widetilde{\mathbf{G}}_{i, j-1}^{+}, \widetilde{\mathbf{G}}_{i, j+1}^{+}-\widetilde{\mathbf{G}}_{i, j}^{+}\right)\end{array}\right.$

$\left\{\begin{array}{l}\widetilde{\mathbf{F}}_{i+\frac{1}{2} R, j}^{-}=\widetilde{\mathbf{F}}_{i+1, j}^{-}-\frac{1}{2} \min \bmod \left(\widetilde{\mathbf{F}}_{i+2, j}^{-}-\widetilde{\mathbf{F}}_{i+1, j}^{-}, \widetilde{\mathbf{F}}_{i+1, j}^{-}-\widetilde{\mathbf{F}}_{i, j}^{-}\right) \\ \widetilde{\mathbf{G}}_{i, j+\frac{1}{2} R}^{-}=\widetilde{\mathbf{G}}_{i, j+1}^{-}-\frac{1}{2} \min \bmod \left(\widetilde{\mathbf{G}}_{i, j+2}^{-}-\widetilde{\mathbf{G}}_{i, j+1}^{-}, \widetilde{\mathbf{G}}_{i, j+1}^{-}-\widetilde{\mathbf{G}}_{i, j}^{-}\right)\end{array}\right.$

where $\widetilde{\mathbf{F}}^{ \pm}$and $\widetilde{\mathbf{G}}^{ \pm}$denote the flux vector splitting according to Steger and Warming's method [22], and the time-marching integration was performed using a second-order accurate Runge-Kutta integration. As to the NND scheme, it was proved to satisfy the dispersion conditions derived by Jiang et al. [23] considering the role of dispersion terms in the modified equation of Warming and Hyett. The conditions require that shock-capturing schemes must have different phrase errors (leading or lagging) on each side of a shock wave in computation to avoid non-physical oscillations near shock waves. Non-oscillatory solutions can be achieved with the scheme without any needs for additional artificial viscosity and free parameters [24]. This characteristic is important to capture fine shock wave structures, which may, otherwise, be smeared with the artificial viscosity.

\section{Validation of numerical methods and codes}

In order to explore the flow physics with numerical CFD technology, the solution validation has to be carried out to demonstrate the reliability of numerical results. Two special cases were chosen because the detailed experimental data is not available for the present study. The first case is a planar CJ detonation propagating in a straight tube to check the detonation model and its ignition simlification. The second is the two-dimensional cellular detonation reflecting with a $20^{\circ}$ wedge to check cellular detonation features. Furthermore, the effect of solution resolution was also evaluated with three different mesh sizes.

In the first case, the planar detonation propagation was simulated at initial conditions of $p_{0}=1.0 \mathrm{~atm}$ and $T_{0}=293.0 \mathrm{~K}$ with $\Delta x=0.05 \mathrm{~mm}$ that is about $12-15$ grids in a half reaction length. The predicted pressure distributions at several instants were plotted in Fig. 3. The pressure spikes experience sharp variations at the beginning of the propagation due to the ignition effect, and reach to a roughly constant level at a distance about $2.0 \mathrm{~cm}$, The statistics of the numerical data showed that the detonation speed is $2836.32 \mathrm{~m} / \mathrm{s}$, and the pressure at the $\mathrm{CJ}$ point is $18.47 \mathrm{~atm}$. The corresponding data calculated with the detailed chemical reaction mechanism are $2845.16 \mathrm{~m} / \mathrm{s}$ for the detonation speed and $18.45 \mathrm{~atm}$ for the CJ pressure, respectively. The discrepancies fall in $0.31 \%$, hence, the reliability of the present chemical reaction model was well demonstrated.

The second case is the detonation reflection and a plenty of experimental results are available for the qualitative validation. In this case, the detonation front consists of two parts, that is, the incident detonation and the Mach stem detonation, which are of different strength, propagate at different speeds and result 


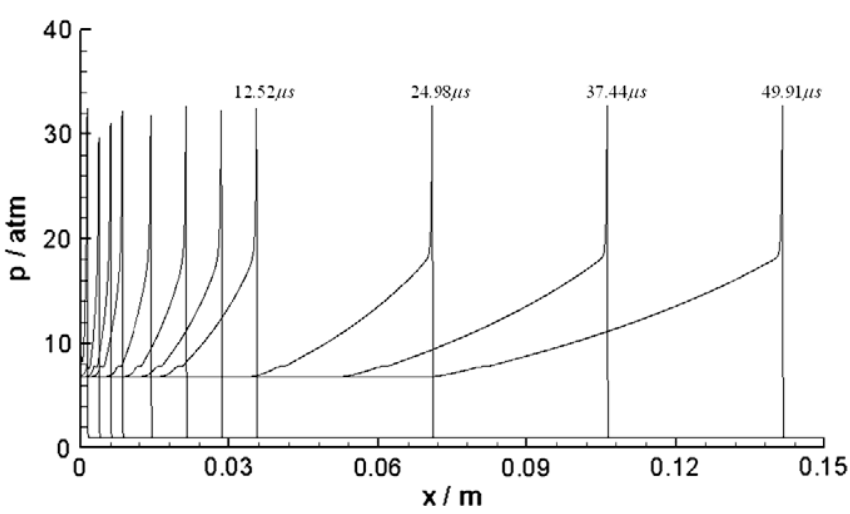

Fig. 3. Pressure distribution at different instants showing planar detonation propagation.

in different cellular cell patterns. The numerical cellular detonation cell pattern and a smoked foil record are presented together in Fig. 4 for comparison. Due to the factors such as turbulence perturbations, flow instability, three-dimensional effects and so on, the experimental cells are more irregular than numerical results. However, it is observable that the wave front near the wedge is overdriven due to the shock reflection, the so-called Mach stem detonation leads to smaller detonation cells behind it, and the quite similar characteristics exists both in the numerical results in Fig. 4a and the smoked foil image in Fig. 4b. Therefore, the reliability of the simulated solution of cellular detonations was also demonstrated.

Mesh resolution tests were carried out by using three different mesh systems as shown in Table 1 . There are about 15-20 grid points in the reaction zone and $50 \times 50$ grid points in a cell for the first test, about 30-40 grid points in the reaction zone and $100 \times 100$ grid points in a cell for the second test, and about 60 100 grid points in the reaction zone, and $200 \times 100$ grid points in a cell for the third test. These numerical tests showed that the det-
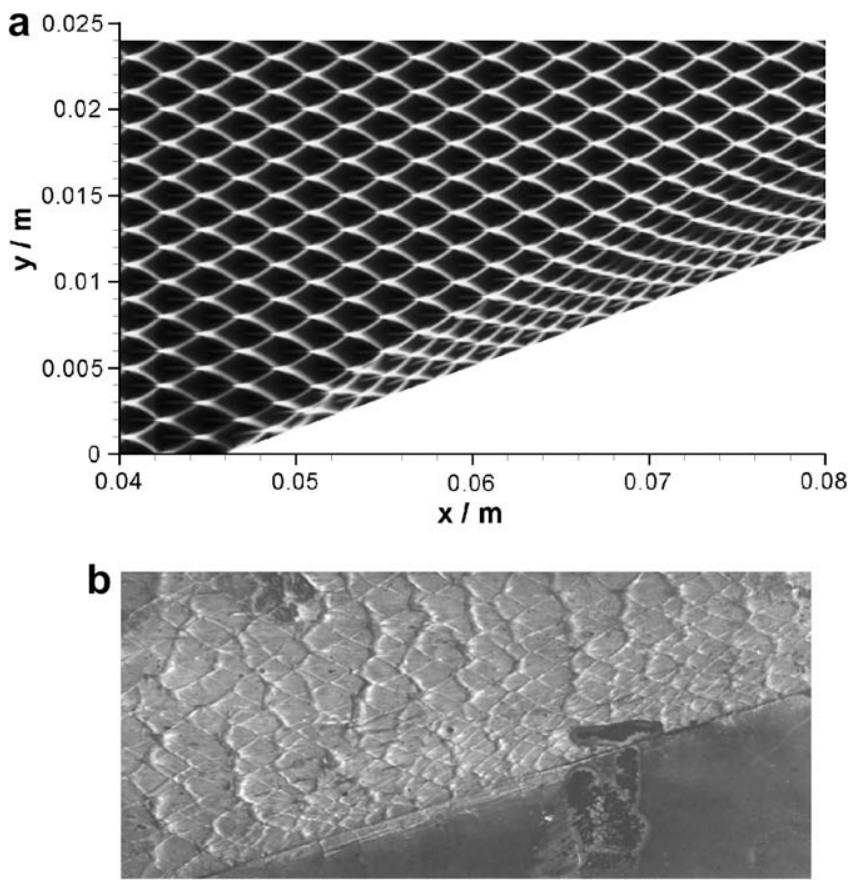

Fig. 4. Detonation reflection from a $20^{\circ}$ wedge with a mixture of $2 \mathrm{H}_{2}+\mathrm{O}_{2}$ at $T_{0}=293.0 \mathrm{~K}$ : (a) Numerical results of detonation cells; (b) Experimental smoke foil photograph [25].
Table 1

Numerical test conditions for mesh resolution evaluation.

\begin{tabular}{lllll}
\hline No. & $\begin{array}{l}\text { Computational } \\
\text { domain }\end{array}$ & $\begin{array}{l}\text { Grids in } \\
\text { total }\end{array}$ & $\begin{array}{l}\text { Grids in reaction } \\
\text { zone }\end{array}$ & $\begin{array}{l}\text { Grids in a } \\
\text { detonation cell }\end{array}$ \\
\hline 1 & $\begin{array}{l}R_{\text {in }}=0.5 \mathrm{~cm}, \\
R_{\text {out }}=15.5 \mathrm{~cm}\end{array}$ & $1500 \times 500$ & $15-20$ & $50 \times 50$ \\
2 & $\begin{array}{l}R_{\text {in }}=0.5 \mathrm{~cm}, \\
R_{\text {out }}=15.5 \mathrm{~cm}\end{array}$ & $3000 \times 1000$ & $30-40$ & $100 \times 100$ \\
3 & $\begin{array}{l}R_{\text {in }}=0.5 \mathrm{~cm}, \\
R_{\text {out }}=8.0 \mathrm{~cm}\end{array}$ & $3000 \times 1000$ & $60-100$ & $200 \times 100$ \\
\hline
\end{tabular}

onation solutions in the second test demonstrated more similar characteristics with experiments, and the numerical results in the third test presented more detonation instabilities and required much more computational time. Therefore, the grid system in the second case was chosen for the further simulation of the transverse wave generation of diverging detonation propagation.

\section{Results and discussions}

The further numerical simulation was carried out and the detonation cell pattern is shown in Fig. 5. The diverging cylindrical cellular detonation is initiated upon rapture of the central hot spot of burnt gas. At the center, the initiation energy manifests itself as a blast wave initiating a slightly overdriven detonation wave, whose overdriven effect quickly vanishes as the wave expands within one detonation cell length. A detonation cell is formed from the trajectory of the frontal triple-shock point where a transverse wave propagates circumferentially. Thus, the detonation cell width defines transverse wave spacing. As the detonation further expands radially, the circumference of the detonation front continuously increases with an increase in detonation cell number. New transverse wave is generated within the scale of a detonation cell as it grows and becomes sufficiently large. Beyond a transition regime of about $4-5 \mathrm{~cm}$, the average dimension of the detonation cell appears to approach a rough constant size. In other words, the average number of transverse waves per unit length along the circumference of the detonation front becomes constant. This means, the growth rate of transverse waves must match the rate of increase in surface area of the detonation front.

The generation of new transverse waves must originate in the instabilities of the detonation front. Carefully examining the cellular pattern shown in Fig. 5, at least four primary modes of the selforganized generation of transverse waves can be identified; these are marked with a cycle in the relevant cellular pattern and numbered as I, II, III and IV, respectively. In the following discussions, theses modes are referred to as the concave front focusing, the

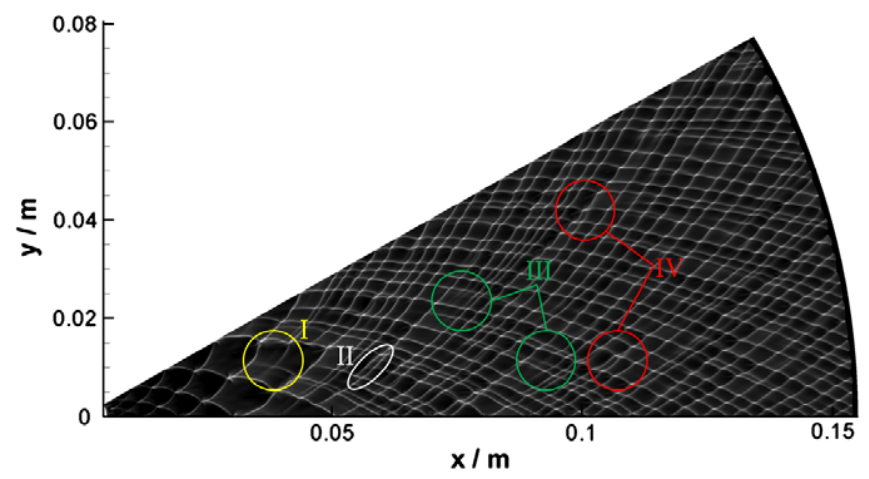

Fig. 5. The numerical cellular pattern of diverging cylindrical detonation with the four modes of the self-organized transverse wave generation, as marked with a cycle, and labeled with I, II, III and IV. 
kinked front evolution, the wrinkled front evolution and the transverse wave merging, respectively.

\subsection{Concave front focusing}

Fig. 6 displays the enlarged cellular pattern showing the region labeled as Mode I in Fig. 5 for the generation of transverse waves

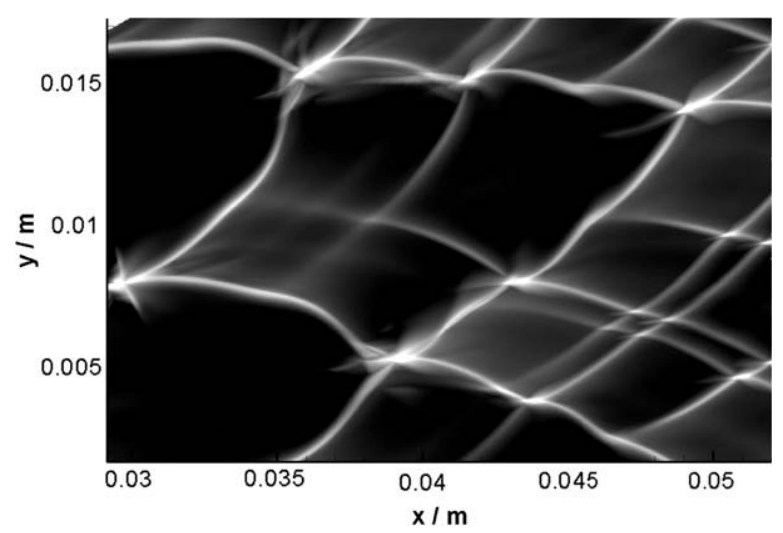

Fig. 6. Enlarged cellular pattern of concave front focusing showing the region labeled as Mode I in Fig. 5.
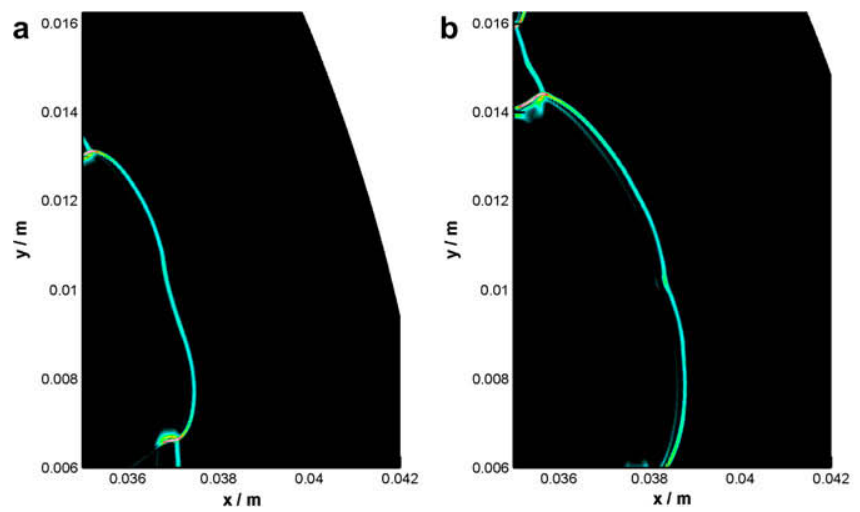

induced by concave front focusing. In the central area of the figure, a big detonation cell is bifurcated into two smaller cells, caused by generation of a pair of new transverse waves at the detonation front associated with the big cell.

The bifurcation process is more clearly illustrated in the density gradient contours in Fig. 7 and a combination of the reaction degree $(\beta)$ distribution in color and the density contour shown in Fig. 8 during the concave front focusing. As the cylindrical detonation expands, a rapid increase in surface area of the detonation front results in a strong flow expansion to weaken the curved wave front. The detonation front decays, the locally-decoupling appears between the chemical reaction and the shock at the weakest wave front location, where the shock becomes slower than its adjacent front and therefore concavely curved (see Fig. 7a). In the concave part of the detonation front, the sparser density contours shown in Fig. 8a indicates the weaker the leading shock, and the reaction zone indicated by the non-red color of $\beta$ distribution becomes wider than the other part of the detonation front. As the concave wave front develops, the local focusing occurs in the unburned flow region between the reaction zone and the leading shock and competes with the effect of flow expansion. The focusing induces a positive feedback loop between the chemical reaction and the shock, leading to a local explosion of the unburned mixture pocket in the originally decoupling zone to turn the concave front into convex (see Fig. 7b and c). Fig. 8c and d shows that the local explosion results in a much denser density distribution and narrower
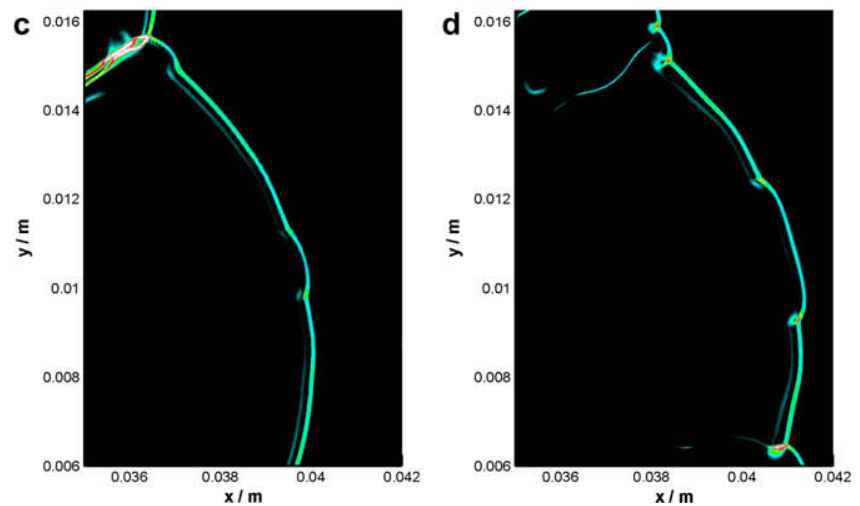

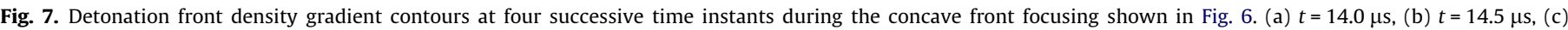
$t=15.0 \mu \mathrm{s}$, (d) $t=15.5 \mu \mathrm{s}$.
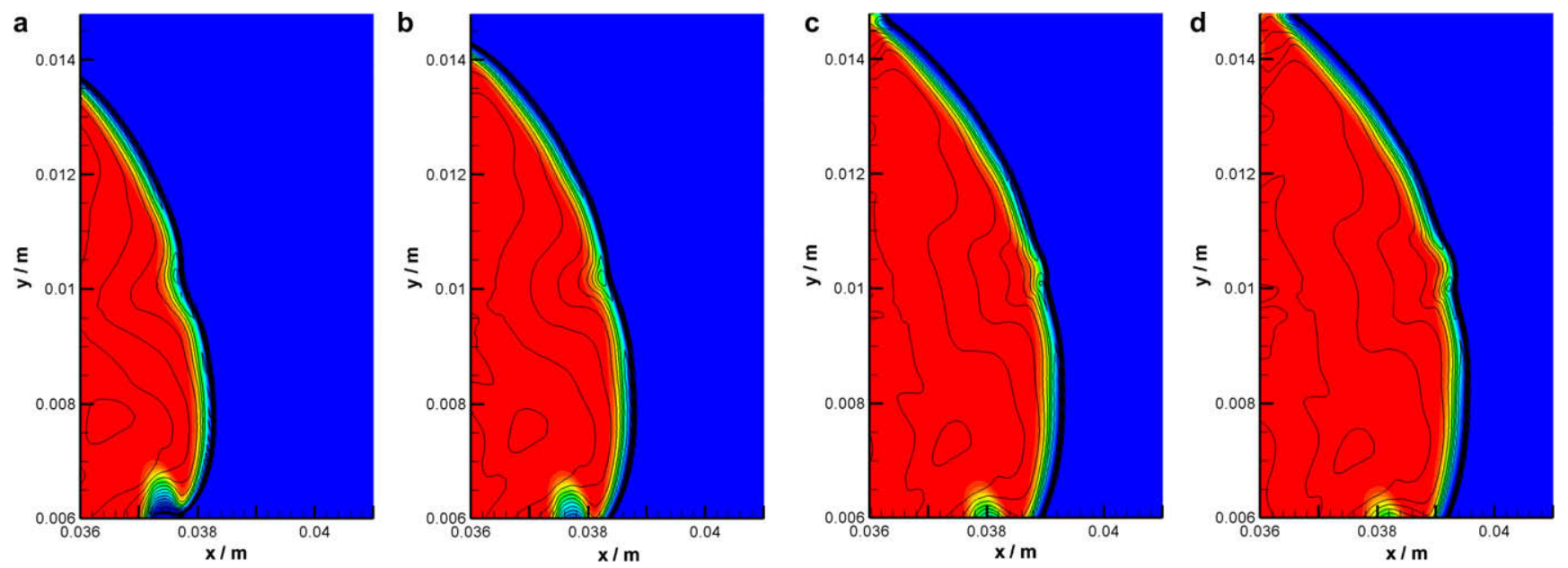

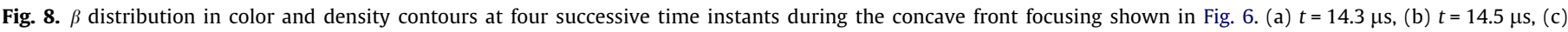
$t=14.7 \mu \mathrm{s}$, (d) $t=14.8 \mu \mathrm{s}$. 
reaction zone than the other part of the detonation front; the local thermodynamic state at the convex region of the detonation front is overdriven. The explosion pushes the leading shock locally outwards, and a new pair of transverse waves are generated and propagate in the opposite directions along the circumference of the detonation front (see Fig. 7d), causing the detonation cell bifurcation.

Fig. 9 schematically shows the underlying mechanism for the concave front focusing and detonation cell bifurcation process. As the flow expands behind the leading shock with diverging detonation front, the middle Mach stem part of the detonation front becomes the weakest and begins to be concave after the Mach stem stretches above a critical length. Within the concave wave front region, both flame front and leading shock act to converge and focus the unburned mixture flow between them (see Fig. 9b in the leading shock coordinate frame). It is these focusing events that lead to the local explosion of the unburned mixture behind the middle of the Mach stem, resulting in the generation of two transverse waves and the bifurcation of original detonation cell when it reaches to its maximum width. The mode of the concave front focusing is mostly observed in the earlier stage of the diverging cylindrical detonation with large front expansion rates, but also appears in the later stage (e.g., location after the marked red circle in Fig. 10).

\subsection{Kinked front evolution}

The second mode is referred to as the kinked front evolution and Fig. 10 displays an enlarged cellular pattern for the region marked as Mode II in Fig. 5. As shown in the marked ellipse of
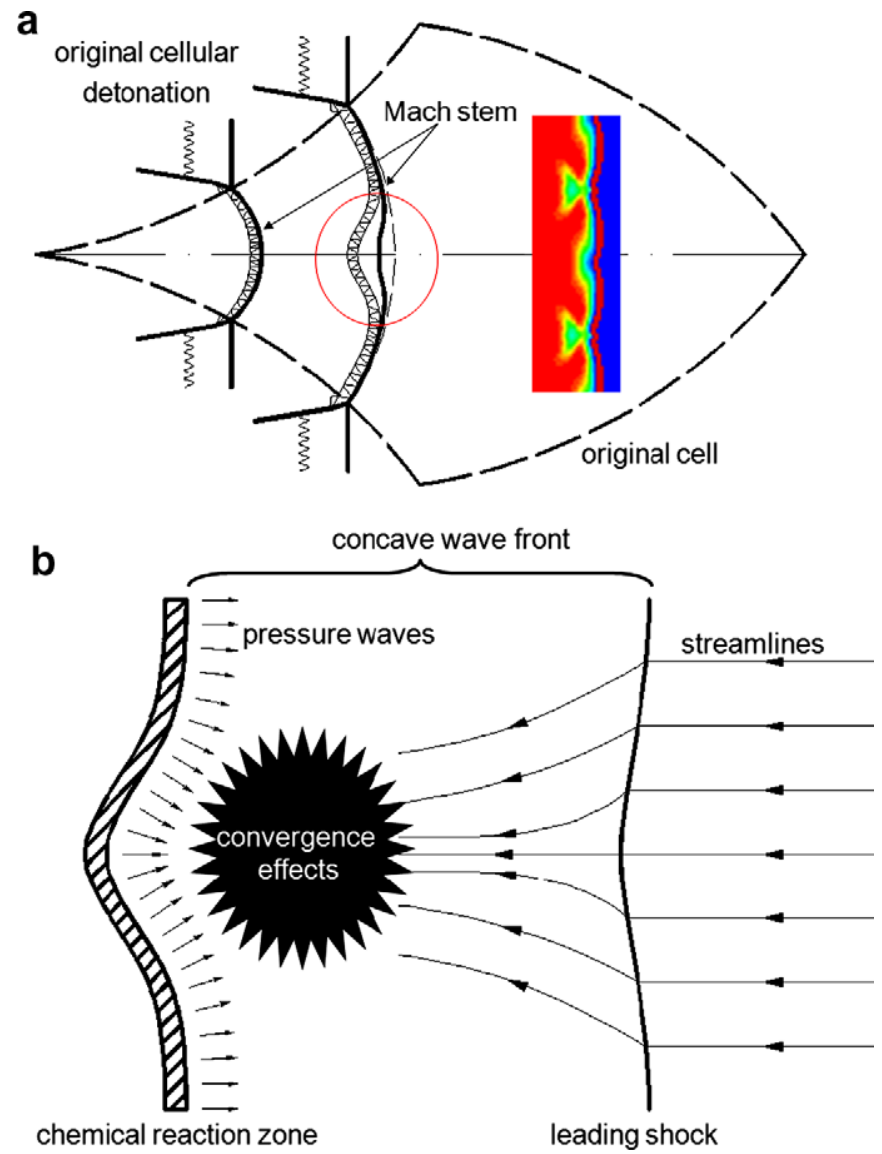

Fig. 9. Schematic of the mechanism for transverse wave generation resulting from concave wave front focusing. (a) concave wave front structure, (b) details of the circle in (a)

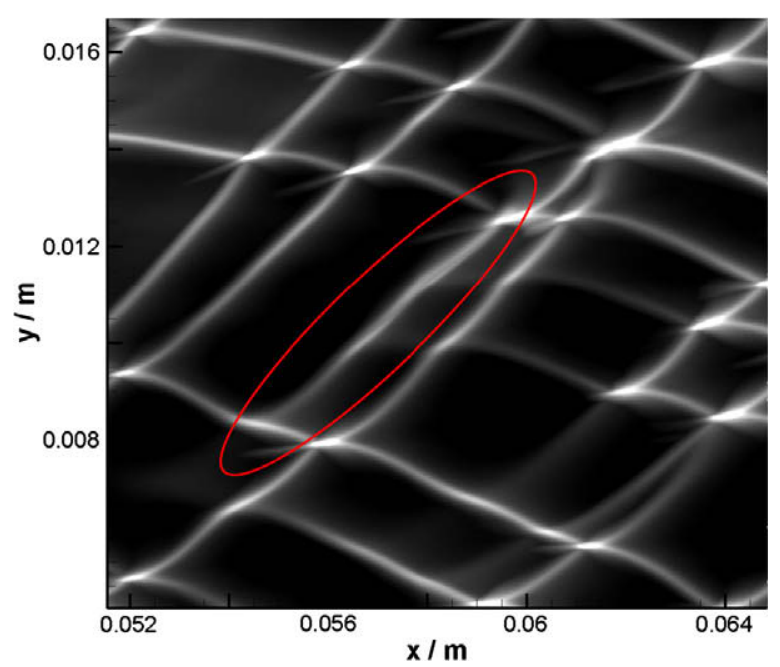

Fig. 10. Enlarged cellular pattern of kinked front evolution showing the region labeled as Mode II in Fig. 5.

Fig. 10, a new triple-point trajectory is generated near the original one; it does not look obvious at its beginning and gets more and more intensified later. This indicates that the transverse wave is born weakly, but gradually amplifies during the propagation. In fact, similar phenomena of transverse wave instability can also be identified in the fine structure corresponding to an overdriven detonation in the vicinity of the triple points near the large bifurcating detonation cell shown in Fig. 6. For the confined marginal or spinning detonation in tubes or channels, a number of experiments in the literature show that these fine-structured transverse waves manifest themselves as the sub-diamond pattern in a band associated with the main triple-point trajectory. Under unconfined cylindrical or spherical geometries, these fine-structured transverse waves may amplify to form source of new transverse waves as the diverging detonation expands and becomes marginal.

In order to elucidate the underlying mechanism for transverse wave generation in this mode, the density gradient contours and a combination of the reaction degree distribution and density contour are plotted in Figs. 11 and 12, respectively, during the process of kinked front evolution. As the diverging detonation expands, the flow instabilities in the transverse direction are developed between the decoupling flame front and leading shock. This leads the detonation front to be deformed and kinked behind a clockwise-traveling transverse wave, as shown in Figs. 11 and $12 \mathrm{a}$ where the kinked front location is pointed with an arrow. The contact interface instability developed in the kinked front mixes the burned and unburned mixture and the subsequent combustion, in turn, amplifies the kinking of the front as illustrated in Figs. $11 \mathrm{~b}$ and $12 \mathrm{~b}$ and $\mathrm{c}$. This cycle progressively repeats, leading to formation of a transverse shock wave that propagates in the unburned mixture in the counter-clockwise direction (see Figs. 12d and $11 \mathrm{c}$ and $\mathrm{d}$ ) and ignites the reaction of the unburned mixture.

\subsection{Wrinkled front evolution}

The Wrinkled front evolution is essentially the weak wrinkled transverse instabilities and often appears at multiple locations on the detonation front within a cell scale. An example is shown in Fig. 13 as an enlarged cellular pattern for the region marked as Mode III in Fig. 5. Fig. 13 shows at least two weak triple-point trajectories identified in the marked circle. The corresponding transverse waves generated are weak at the beginning and are gradually intensified later, leading to the development of three cells from the original cell. 

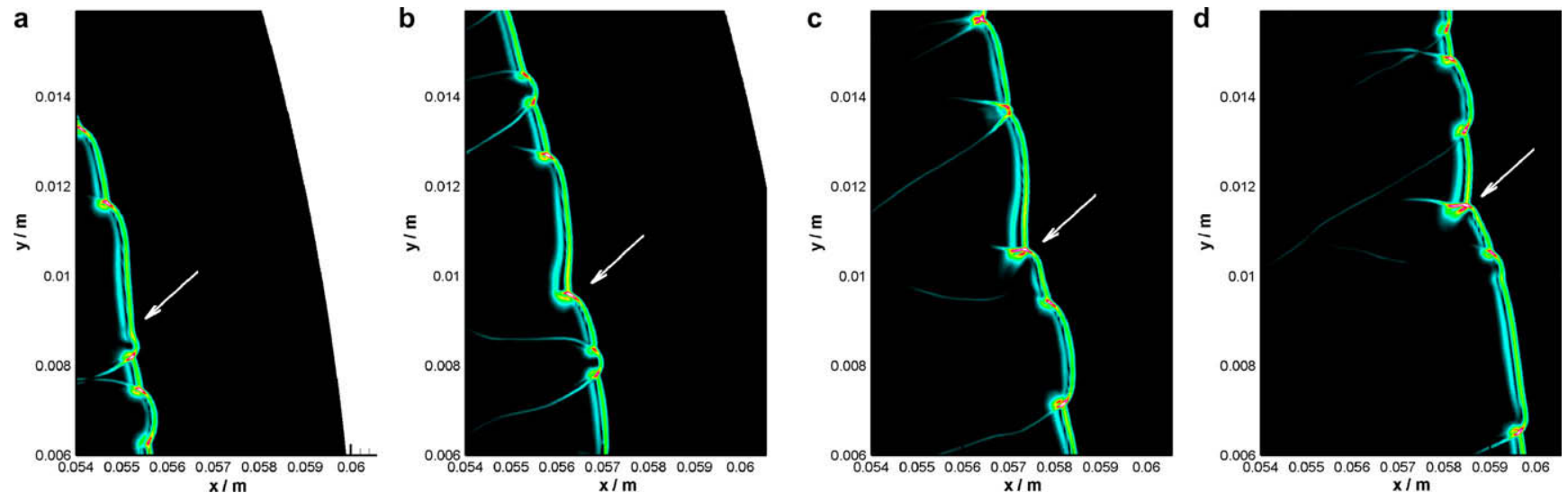

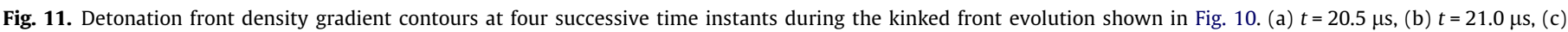
$t=21.5 \mu \mathrm{s}$, (d) $t=22.0 \mu \mathrm{s}$.
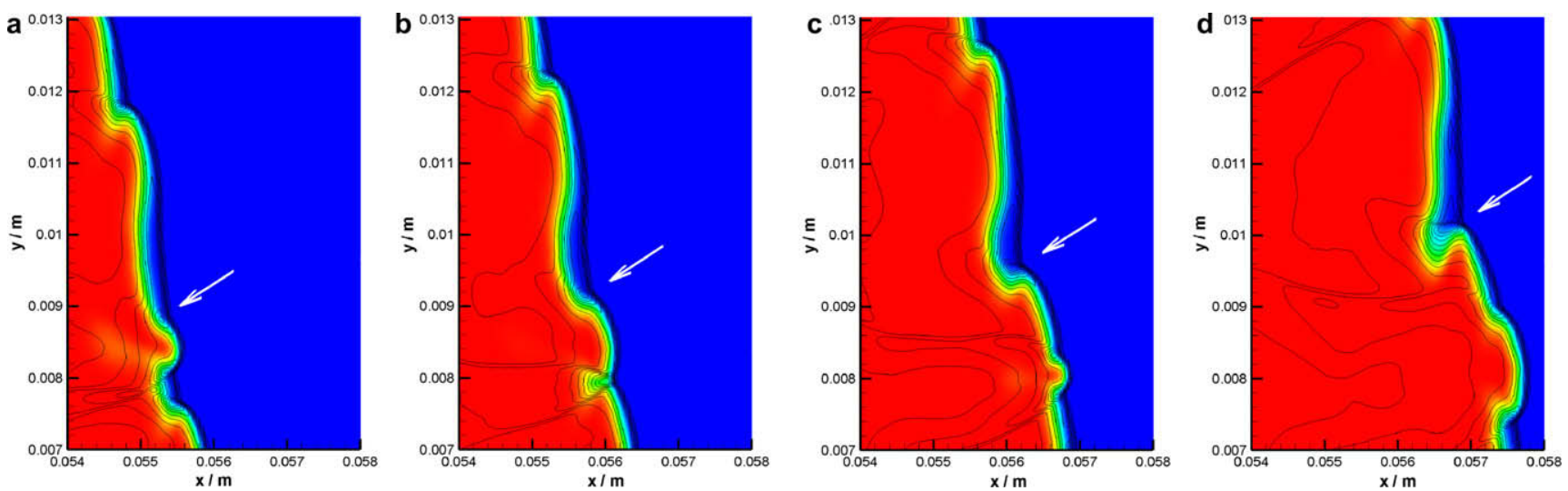

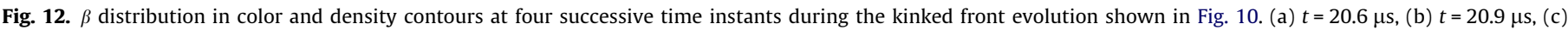
$t=21.0 \mu \mathrm{s}$, (d) $t=21.3 \mu \mathrm{s}$.

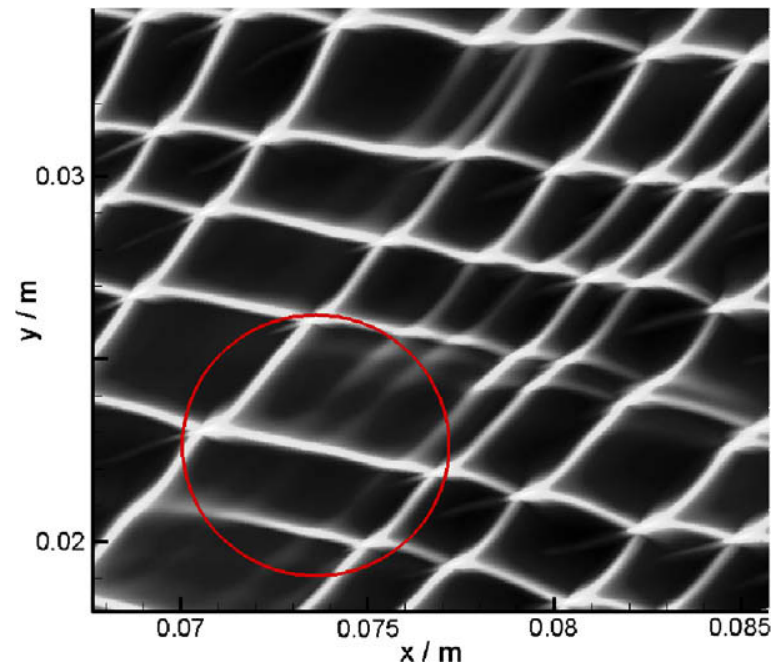

Fig. 13. Enlarged cellular pattern of wrinkled front evolution showing the region labeled as Mode III in Fig. 5.

This kind of transverse wave instability development is more clearly illustrated in the reaction degree distribution in color and density contour, as shown in Fig. 14. As the cylindrical detonation expands, the detonation front becomes unstable due to the sensitivity of the chemical reaction to the flow expansion and perturba- tions behind the shock front. Three weakly wrinkled instabilities are developed in the subsequent times as shown in Fig. 14b and $c$ at the local detonation front between two opposite-propagating transverse waves. This makes this part of the detonation front to appear wrinkled geometry, from which new transverse waves are developed. The evolution of the weak instabilities of the detonation front indicates the sensitivity of the chemical reaction at a critical sate to flow or wave disturbances. Acceleration loop of the nonlinear chemical reaction coherent with the leading shock can lead to a significant growth of the front instability.

\subsection{Transverse wave merging}

Fig. 15 provides the enlarged cellular pattern showing the region labeled as Mode IV in Fig. 5 for the merging of transverse waves. Initially, three triple-point trajectories associated with three transverse waves are identified as $S_{1}, S_{2}$ and $S_{3}$ where $S_{3}$ closely follows $S_{2}$. Collision of $S_{1}$ and $S_{2}$ results in two reflected transverse waves associated with triple points $S_{1}^{\prime}$ and $S_{2}^{\prime}$. Upon interacting with the strong reflected transverse wave associated with $S_{2}^{\prime}$, the transverse wave associated with $S_{3}$ becomes weaker and finally diminishes. In other words, the transverse wave associated with $\mathrm{S}_{3}$ is engulfed in the rear flow of the reflected transverse shock associated with $S_{2}^{\prime}$ and the two original cells merge into one.

Fig. 16 further illustrates the physical process of the self-merging of transverse waves using the density distributions at successive time instants. In Fig. 16a and b, two transverse waves, marked with $\mathrm{A}$ and $\mathrm{B}$, move towards each other, while a third 

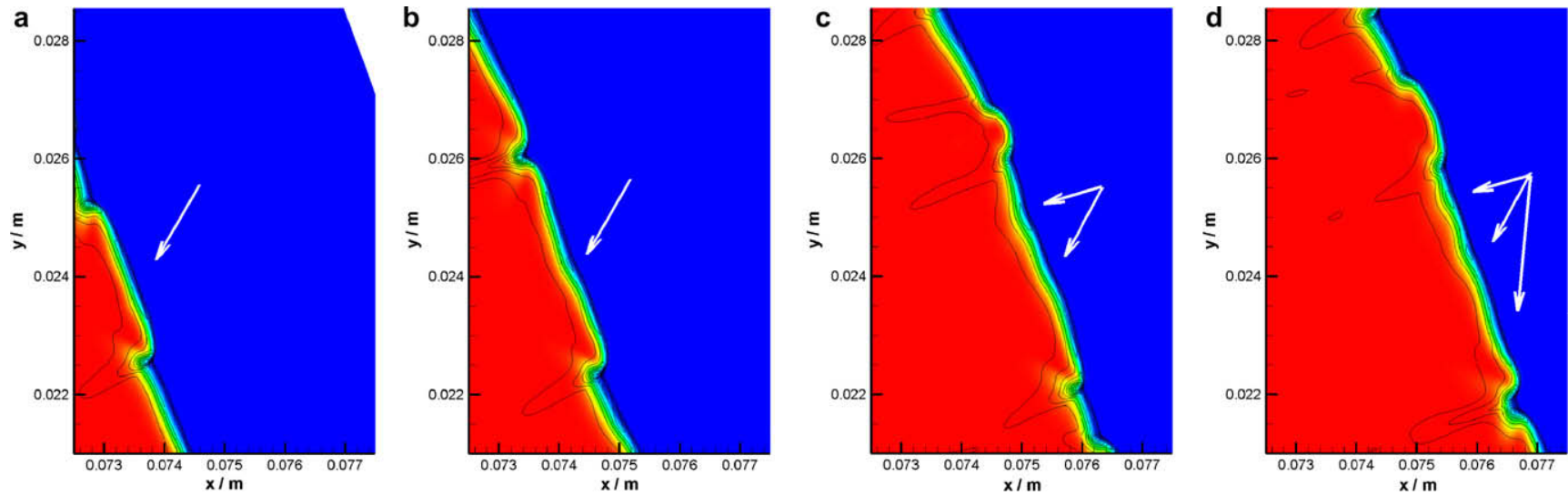

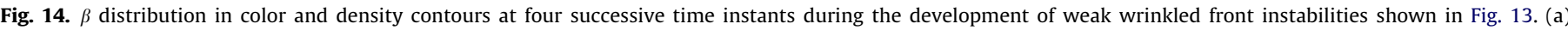
$t=28.2 \mu \mathrm{s}$, (b) $t=28.5 \mu \mathrm{s}$, (c) $t=28.9 \mu \mathrm{s}$, (d) $t=29.1 \mu \mathrm{s}$.

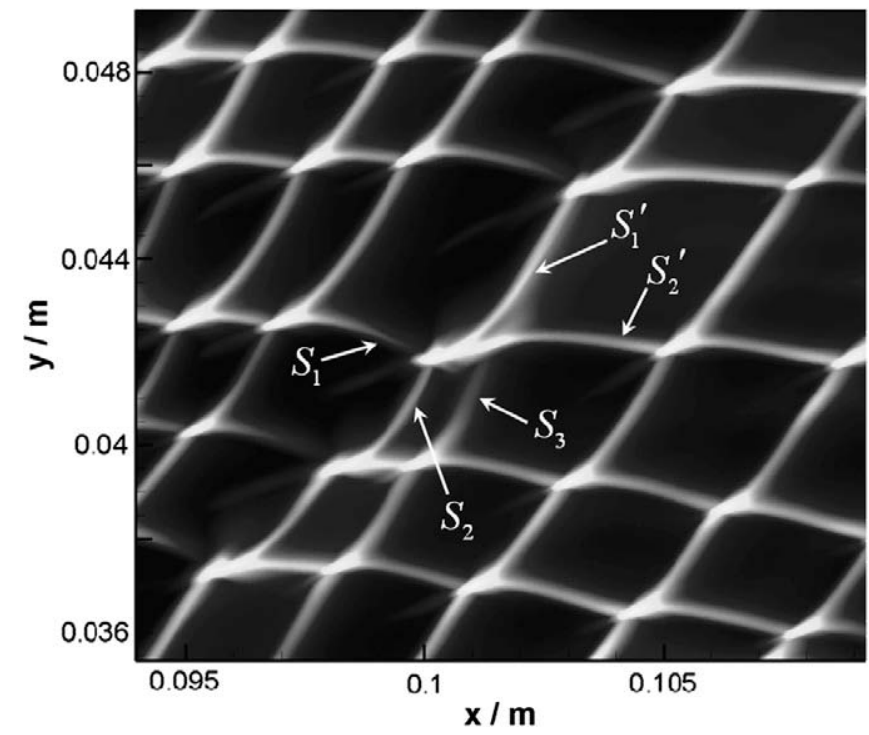

Fig. 15. Cellular cell pattern, referred to as the self-merging of cellular cells, with an enlarged view showing the region marked with a circle and labeled as Mode IV in Fig. 5.

transverse wave referred to as $C$ follows transverse wave $B$ in the same direction. After collision of two transverse waves $\mathrm{A}$ and $\mathrm{B}$, the initially reflected transverse wave $B^{\prime}$ is strongly overdriven as it encounters the coming transverse wave $C$ (shown in Fig. 16c). Upon their interaction, the transmitted wave propagates into the burned gas left behind transverse wave B' and therefore gains little chemical energy release to sustain itself. Thus, the transmitted wave becomes weaker and finally diminishes as shown in Fig. 16d. At the end of this merging process, only a pairs of transverse waves $A^{\prime}$ and $B^{\prime \prime}$ exist.

\section{Conclusion}

In unconfined cylindrical and spherical geometries, the propagation mechanism of a diverging detonation wave is likely controlled by the transverse wave instabilities. As the detonation expands with radius, the surface area of the detonation front continuously increases with an increase in number of cells. New transverse waves must be generated at the detonation front to maintain the propagation of the detonation with a transverse wave spacing asymptotically towards a constant size (an inherent length scale of the chemically reactive system) while the surface area of the detonation front continuously increases. Thus, the growth rate of transverse waves must match the rate of increase in surface area of the detonation front. In this paper, the origin and mechanisms for the growth of transverse waves at the detonation front have been studied for the diverging cylindrical detonation, using twodimensional unsteady Euler equations for numerical simulations.

Several underlying mechanisms for the self-organized generation of transverse waves have been identified and referred to as: the concave front focusing, the kinked front evolution and the
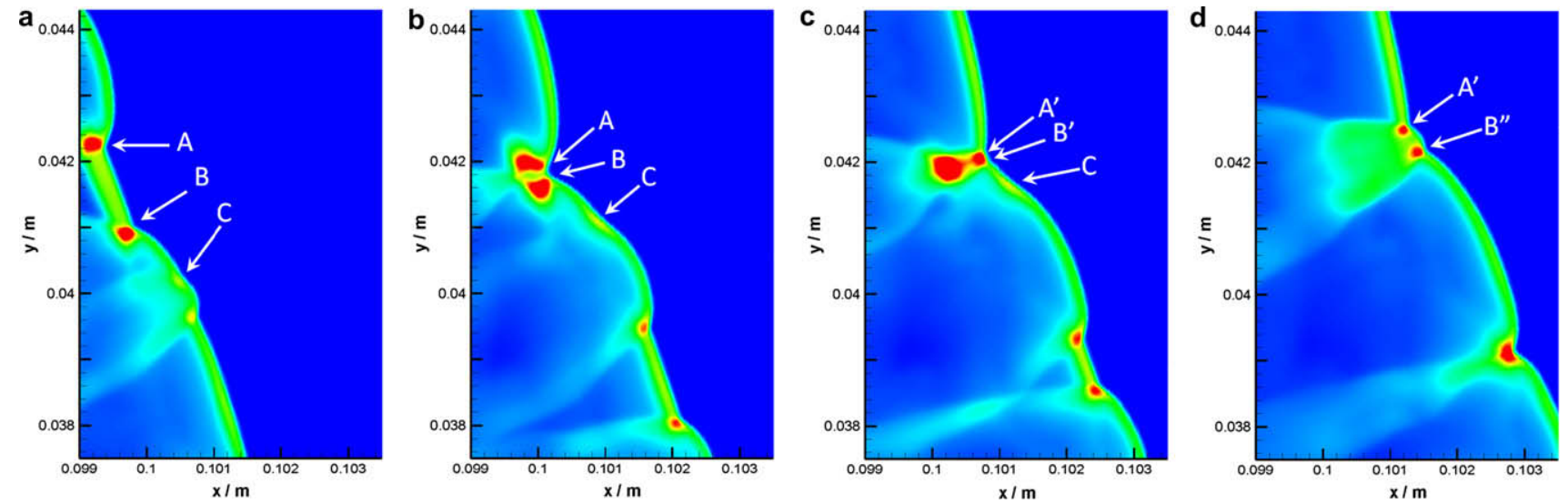

Fig. 16. Density distributions showing the cellular cell self-merging at four successive time instants. (a) $t=39.3 \mu \mathrm{s}$, (b) $t=39.6 \mu \mathrm{s}$, (c) $t=39.8 \mu \mathrm{s}$, (d) $t=40.0 \mu \mathrm{s}$. 
wrinkled front evolution, respectively. In the mechanism of concave front focusing, the weakest part of the Mach stem in the detonation front becomes concaved due to the expansion decoupling of the reaction with the Mach stem shock as the cylindrical detonation expands and the circumference of the detonation front increases. The locally concaved geometry of the detonation front leads to the wave focusing and explosion in the unburned pocket of the mixture between the flame front and the leading shock, resulting in a pair of new transverse waves at the front. The concave front focusing usually takes place where a large rate of increase in front surface area occurs. In the mechanism of kinked front evolution, the local flow instabilities in the transverse direction of the detonation front are developed between the decoupling flame front and leading shock, leading to kinking deformation of the detonation front. The contact interface instability developed in the kinked front mixes the burned and unburned mixture and the subsequent combustion amplifies the front kinking progressively to form a transverse shock wave propagating in the unburned mixture. The wrinkled front evolution is essentially the weak kinked transverse instabilities at multiple locations on the detonation front within a detonation cell scale. The evolution of the weak instabilities of the detonation front indicates the sensitivity of the chemical reaction at a critical sate to flow or wave disturbances. While the above mechanisms generate new transverse waves, self-adjustment in eliminating extra weak transverse waves to maintain the constant transverse wave spacing on the enlarging detonation front also occurs by the interaction of the two transverse waves. After the interaction, the weaker transverse wave propagates in the combustion products and diminishes due to a lack of further energy release support.

Thus, under the sufficient initiation energy, the transverse wave instabilities, intrinsic to the chemical reactive mixture, compete with the rate of increase in surface area of the diverging detonation front and lead to a growth of transverse waves to maintain a constant transverse wave spacing at the expanding detonation front. Due to the limitation of the mesh resolution, this study provides a qualitative description and other types of transverse wave generation are not excluded. More investigation using higher mesh resolution would also be required in establishing quantitative dependence of various mechanisms of transverse wave generation on the rate of increase in surface area of the diverging detonation front.

\section{Acknowledgments}

The present work is supported by the National Natural Science Foundation of China under Grant No. 10632090. The first author would like to thank Prof. John Lee in McGill Univer- sity, Montreal, Canada for his support when the author visiting his laboratory.

\section{References}

[1] E. Jouguet, La Mecanique des Explosifs, Paris Ed. Doin, 1917, pp. 359-366.

[2] R. Courant, K.O. Friedrich, Supersonic Flows and Shock Waves, Interscience Pub. No. 4, 1950, 430 pp.

[3] G.I. Taylor, The formation of a blast wave by very intense explosion, I. Theoretical discussion, Proc. Roy. Soc. Lond. A 201 (1950) 159-174.

[4] N. Manson, F. Ferrie, Contribution to the study of spherical detonation waves, in: Proc. of the 4th Symposium (International) on Combustion, Williams \& Wilkins, Baltimore, MD, 1953, 486 pp.

[5] J.H.S. Lee, R. Knystautas, G.G. Bach, Theory of explosion, Scientific Report AFOSR 69-3090 TR, 1969, pp. 13-31.

[6] W.W. Wood, J.G. Kirkwood, Diameter effect in condensed explosives: the relation between velocity and radius of curvature of the detonation wave, J. Chem. Phys. 22 (1954) 1920-1924.

[7] Y.B. Zeldovich, A.S. Kompaneets, Theory of Detonation, Academic Press, London, 1960. pp. 133-205.

[8] L. He, P. Clavin, On the direct initiation of gaseous detonations by an energy source, J. Fluid Mech. 277 (1994) 227-248.

[9] J. Yao, D.S. Stewart, On the dynamics of multi-dimensional detonation, J. Fluid Mech. 309 (1996) 225-275.

[10] C.A. Eckett, J.J. Quirk, J.E. Shepherd, The role of unsteadiness in direct initiation of gaseous detonations, J. Fluid Mech. 421 (2000) 147-183.

[11] H.D. Ng, J.H.S. Lee, Direct initiation of detonation with a multi-step reaction scheme, J. Fluid Mech. 476 (2003) 179-211.

[12] S.D. Watt, G.J. Sharpe, Linear and nonlinear dynamics of cylindrically and spherically expanding detonation waves, J. Fluid Mech. 533 (2005) 329-356.

[13] C. Wang, Z. Jiang, Numerical simulation of the cellular structure evolution in cylindrically diverging detonation, in: Proc. of the 26th International Symposium on Shock Waves, Goettingen, Germany, 15-20 July, 2007.

[14] G. Han, Z. Jiang, C. Wang, F. Zhang, Cellular cell bifurcation of cylindrical detonations, Chin. Phys. Lett. 25 (6) (2008) 2125-2127.

[15] R.I. Soloukhin, Diverging wave in spin detonation, Izv. Akad. Nauk. SSSR, OTN, Mekhan 6 (1959) 145.

[16] R.I. Soloukhin, Shock Waves and Detonations in Gases, State Publishing House, Moscow, 1963. English Translation, Mono Book Corp., 1966, pp. 138-147.

[17] S.M. Kogarko, V.V. Adushkin, A.G. Lyamin, Nauchno teknicheskie problemy goreniya i Vzryva, vol. 2, 1965, p. 22.

[18] D.C. Bull, J.E. Elsworth, G. Hooper, C.P. Quinnt, A study of spherical detonation in mixtures of methane and oxygen diluted by nitrogen, J. Phys. D: Appl. Phys. 9 (1976) 191-200.

[19] M. Sichel, N. Tonello, E. Oran, D. Jones, A two-step kinetics model for numerical simulation of explosions and detonations in $\mathrm{H}_{2}-\mathrm{O}_{2}$ mixture, Proc. Roy. Soc. Lond. A 458 (2001) 49-82.

[20] B. McBride, M. Zehe, S. Gordon, NASA Glenn coefficients for calculating thermodynamics properties of individual species, NASA/TP 211556, 2002.

[21] H. Zhang, Non-oscillatory and non-free-parameter dissipation difference scheme, Acta Aerodyn. Sin. 6 (1988) 143-165.

[22] J.L. Steger, R.F. Warming, Flux vector splitting of the invicid gasdynamics equations with applications to finite difference methods, J. Comp. Phys. 40 (1981) 263-293.

[23] Z. Jiang, K. Takayana, Y.S. Chen, Dispersion conditions for non-oscillatory shock capturing schemes and its applications, Comput. Fluid Dynam. J. 4 (1995) 37150.

[24] Z. Jiang, On the dispersion-control principles for non-oscillatory shock capturing schemes, ACTA Mech. Sin. 20 (1) (2004) 1-15.

[25] G.O. Thomas, R.L. Williams, Detonation interaction with wedges and bends, Shock Wave 11 (2002) 481-492. 